\title{
Some connections between almost periodic and periodic discrete Schrödinger operators with analytic potentials
}

\author{
Mira Shamis ${ }^{1}$
}

\begin{abstract}
We study discrete Schrödinger operators with analytic potentials. In particular, we are interested in the connection between the absolutely continuous spectrum in the almost periodic case and the spectra in the periodic case. We prove a weak form of a precise conjecture relating the two.

We also bound the measure of the spectrum in the periodic case in terms of the Lyapunov exponent in the almost periodic case.

In the proofs, we use a partial generalization of Chambers' formula. As an additional application of this generalization, we provide a new proof of Herman's lower bound for the Lyapunov exponent.
\end{abstract}

Mathematics Subject Classification (2010). 9A70, 47B39.

Keywords. Discrete Schrödinger operators, almost periodic potential, absolutely continuous spectrum, periodic approximation.

\section{Introduction}

In this paper we consider discrete Schrödinger operators of the form

$$
\left[H_{\alpha, \theta} \psi\right](n)=\psi(n+1)+\psi(n-1)+V_{\alpha, \theta}(n) \psi(n), \quad \psi \in \ell^{2}(\mathbb{N}),
$$

where we formally set $\psi(0)=0$. The potential

$$
V_{\alpha, \theta}(n)=f(2 \pi \alpha n+\theta), \quad \alpha \in \mathbb{R}, 0 \leq \theta<2 \pi,
$$

is constructed from a function $f$ which is periodic of period $2 \pi$, and analytic in a strip $\{z \in \mathbb{C}|| \operatorname{Im} z \mid \leq \eta\}$. We also assume that $f$ is real on $\mathbb{R}$; in this case the operator is self-adjoint.

As a primary example, one may think of the special case when $f$ is a trigonometric polynomial

$$
P(\theta)=\sum_{k=-d}^{d} a_{k} \exp (i k \theta),
$$

\footnotetext{
${ }^{1}$ Supported by NSF under agreement DMS-0635607.
} 
where we assume that $a_{-k}=\bar{a}_{k}$ for $-d \leq k \leq d$.

If $\alpha \in \mathbb{Q}$, the potential is periodic, and the spectrum $\sigma(p / q, \theta)$ of $H_{\alpha, \theta}$ is a union of $q$ closed intervals. ${ }^{1}$ Denote

$$
S_{-}\left(\frac{p}{q}\right)=\bigcap_{0 \leq \theta<2 \pi} \sigma\left(\frac{p}{q}, \theta\right) .
$$

If $\alpha \notin \mathbb{Q}$, the operator is almost periodic. In this case the spectrum does not depend on $\theta$ (by Pastur's theorem, see, e.g., [5], Theorem 9.2). We shall mainly be interested in the set

$$
\mathcal{A}(\alpha)=\{E \mid \bar{\gamma}(E, \alpha)=0\}
$$

where the Lyapunov exponent $\bar{\gamma}$ (see Section 2.1 for the definition) vanishes. According to the Ishii-Kotani-Pastur Theorem 9.13 in [5], $\mathcal{A}(\alpha)$ is an essential support of the absolutely continuous spectrum of $H_{\alpha, \theta}$ for any $0 \leq \theta<2 \pi$, that is, a minimal (up to Lebesgue measure zero) set which supports the absolutely continuous part of the spectral measure.

We are interested in the connection between the set $\mathcal{A}(\alpha)$ for irrational $\alpha$ and the spectra $\sigma(p / q, \theta)$ of the periodic operators corresponding to $p / q$ that are close to $\alpha$. Apart from the intrinsic interest, this connection is often used to study almost periodic operators via their periodic approximations.

This paper is motivated by the following conjecture, which we learned from Y. Last.

Conjecture. For any $\alpha \notin \mathbb{Q}, \mathcal{A}(\alpha)=\lim _{p / q \rightarrow \alpha} S_{-}(p / q)$, that is

$$
\underset{\frac{p}{q} \rightarrow \alpha}{\limsup } S_{-}\left(\frac{p}{q}\right)=\bigcap_{\delta>0} \bigcup_{\left|\frac{p}{q}-\alpha\right|<\delta} S_{-}\left(\frac{p}{q}\right)
$$

and

$$
\liminf _{\frac{p}{q} \rightarrow \alpha} S_{-}\left(\frac{p}{q}\right)=\bigcup_{\delta>0} \bigcap_{\left|\frac{p}{q}-\alpha\right|<\delta} S_{-}\left(\frac{p}{q}\right)
$$

coincide (at least, up to Lebesgue measure zero) with one another and with $\mathcal{A}(\alpha)$.

We remark that equality modulo sets of measure zero would be sufficient for most of the applications.

The intuition is roughly as follows. If $\alpha \notin \mathbb{Q}$ is very close to $p / q$, then, for any $\theta_{1}$ and $\theta_{2}$, one can find long pieces of $V_{\alpha, \theta_{1}}$ that are close to long repetitions of the period of $V_{p / q, \theta_{2}}$. Therefore, if $E \notin \sigma\left(p / q, \theta_{2}\right)$, the potential $V_{\alpha, \theta_{1}}$ contains

\footnotetext{
${ }^{1}$ Formally, the spectrum of a periodic operator on the half-line also includes $q-1$ simple eigenvalues. We abuse the notation and denote by $\sigma(p / q, \theta)$ the spectrum without these eigenvalues, which is actually the essential spectrum of the operator.
} 
"barriers" which prohibit conductivity at energy $E$, namely, $E$ is outside the absolutely continuous spectrum of $H_{\alpha, \theta_{1}}$. Vice versa, if $E \in \sigma(p / q, \theta)$ for all $\theta$ and all $p / q$ sufficiently close to $\alpha$, the Lyapunov exponent $\bar{\gamma}(E, \alpha)$ should be zero (since $\bar{\gamma}(E, p / q, \theta)=0$ for every $\theta)$.

As additional evidence for the conjecture, we remark that (modulo Lebesgue measure zero) it holds for the almost Mathieu operator, which corresponds to $f(\theta)=$ $\lambda \cos \theta$ for $\lambda \neq 0$ (that is, $d=1$ in (1.1)). This follows from the known results about the measure and the structure of the spectrum for the almost Mathieu operator, see, e.g., the review of Last [13].

It appears that one direction of the conjecture can be derived directly from the result of Bourgain and Jitomirskaya [3]. Namely, the following holds.

Theorem 1.1. For any $\alpha \notin \mathbb{Q}$,

$$
\mathcal{A}(\alpha) \supset \limsup _{\frac{p}{q} \rightarrow \alpha} S_{-}\left(\frac{p}{q}\right)
$$

Remark. A related result was proved by Last in [12] for a certain set of $\alpha$-s of full Lebesgue measure.

In the other direction, we have only been able to prove a weaker result.

Theorem 1.2. For $\varepsilon>0$, denote

$$
S_{-}\left(\frac{p}{q}, \varepsilon\right)=\bigcap_{\theta}\left\{E \mid \operatorname{dist}\left(E, \sigma\left(\frac{p}{q}, \theta\right)\right)<\varepsilon\right\},
$$

where

$$
\operatorname{dist}(E, K)=\inf _{E^{\prime} \in K}\left|E-E^{\prime}\right|
$$

Then for any $\alpha \notin \mathbb{Q}$

$$
\mathcal{A}(\alpha) \subset \bigcap_{\varepsilon>0} \liminf _{\frac{p}{q} \rightarrow \alpha} S_{-}\left(\frac{p}{q}, \varepsilon\right) .
$$

We also prove an estimate for the average measure of $\sigma(p / q, \theta)$ in terms of the Lyapunov exponent.

Theorem 1.3. There exists a number $d=d(f)$ such that the following holds. Fix $\alpha \notin \mathbb{Q}$. For any $\varepsilon>0$ there exists $\delta>0$ such that for $|p / q-\alpha|<\delta$ and for any $E$ for which $\bar{\gamma}(E, \alpha)>\varepsilon$

$$
\left|\left\{\theta \mid E \in \sigma\left(\frac{p}{q}, \theta\right)\right\}\right| \leq C \exp \left[-\frac{q}{2 d}(\bar{\gamma}(E, \alpha)-\varepsilon)\right] .
$$


In particular, for any compact $I \subset \mathbb{R}$,

$$
\begin{aligned}
\int\left|\sigma\left(\frac{p}{q}, \theta\right) \cap I\right| d \theta & \leq C \int_{I} \exp \left[-\frac{q}{2 d}(\bar{\gamma}(E, \alpha)-\varepsilon)_{+}\right] d E \\
& \leq C|I| \exp \left[-\frac{q}{2 d}\left(\min _{E \in I} \bar{\gamma}(E, \alpha)-\varepsilon\right)_{+}\right] .
\end{aligned}
$$

Remark. In the case of trigonometric potential (1.1) one may take $d$ to be the degree of $P$.

Here and in the sequel $C>0$ stands for a universal constant the value of which may change from line to line.

Note that, for "most" values of $\theta$ the inequality (1.2) gives an upper bound for the measure of the spectrum $\sigma(p / q, \theta)$ which is exponentially small in $q$ in the region of positive Lyapunov exponent. We do not know whether such a bound is true for all values of $\theta$.

The paper is built as follows. In Section 2, we collect the preliminaries we need in the sequel. In particular, in Section 2.2 we use Avila's argument to effectively reduce the problem to the case of trigonometric polynomials, and in Section 2.3 we state two Remez-Turán type inequalities for trigonometric polynomials (due to Erdélyi and Nazarov) which are an important ingredient in the proof of the results. In Section 2.4 we prove a convenient formula for the Lyapunov exponent, and cite a corollary of the Combes-Thomas estimate.

Section 3 contains several facts which can be seen as partial generalizations of Chambers' formula [4], which was originally proved for the almost Mathieu operator. These form the main component in the proofs of Theorems 1-3, which appear in Section 4. These facts are probably known to specialists; we include the proofs for the convenience of the reader.

Finally, in Section 5 we show how Herman's lower bound [9] on the Lyapunov exponent

$$
\bar{\gamma}(E, \alpha) \geq \ln _{+}\left|a_{d}\right|
$$

for trigonometric potentials (1.1) can be easily recovered using the mechanism of this paper. The argument also shows that $S_{-}(p / q)$ is empty for sufficiently large $q$ if $\left|a_{d}\right|>1$. Note that if the conjecture were true, this would follow immediately from (1.3). For now, we prove this separately, to provide additional support for the conjecture.

Acknowledgment. I thank Sasha Sodin for very pleasant and insightful discussions, and, in particular, for referring me to the inequalities of Erdélyi and Nazarov. I thank Tom Spencer for suggesting to use the Combes-Thomas estimate instead of the bound which appeared in an early draft of this paper. I thank Svetlana Jitomirskaya for helpful comments on an early version of this paper, and for suggesting to apply Avila's argument [1] to extend the results to general analytic potentials. 


\section{Preliminaries}

2.1. Transfer matrices. Let $H$ be a discrete Schrödinger operator with real bounded potential $V$,

$$
[H \psi](n)=\psi(n+1)+\psi(n-1)+V(n) \psi(n) .
$$

The one-step transfer matrices $T_{n}(E)$ are defined as

$$
T_{n}(E)=\left(\begin{array}{cc}
E-V(n) & -1 \\
1 & 0
\end{array}\right),
$$

and the $n$-step transfer matrix $\Phi_{n}(E)$ is defined as

$$
\Phi_{n}(E)=T_{n}(E) \cdots T_{2}(E) T_{1}(E) .
$$

Set $\Delta_{n}(E)=\operatorname{tr} \Phi_{n}(E)$ (where tr stands for the trace); this is a real monic polynomial of degree $n$. If the operator is periodic of period $q$, the polynomial $\Delta=\Delta_{q}$ is called the discriminant of $H$.

For our operator $H_{\alpha, \theta}$, we denote the $n$-step transfer matrix by $\Phi_{n}(E, \alpha, \theta)$, and $\Delta_{n}$ by $D_{n}(E, \alpha, \theta)$. For any $n \geq 1, D_{n}$ is an analytic function of $\theta$. In the special case (1.1), it is a trigonometric polynomial in $\theta$ of degree $n d$.

The Lyapunov exponent $\gamma(E, \alpha, \theta)$ is defined by

$$
\gamma(E, \alpha, \theta)=\lim _{n \rightarrow \infty} \frac{1}{n} \ln \left\|\Phi_{n}(E, \alpha, \theta)\right\| .
$$

According to the Furstenberg-Kesten theorem [7], the limit exists for almost every $\theta$. If $\alpha \notin \mathbb{Q}$, the Lyapunov exponent does not depend on $\theta$. In general, it is convenient to define

$$
\bar{\gamma}(\alpha, E)=\lim _{n \rightarrow \infty} \frac{1}{2 \pi n} \int_{0}^{2 \pi} \ln \left\|\Phi_{n}(E, \alpha, \theta)\right\| d \theta .
$$

For irrational $\alpha, \bar{\gamma}(E, \alpha)=\gamma(E, \alpha, \theta)$.

2.2. Approximation of the discriminant by a trigonometric polynomial. In the general case, $D_{n}(E, \alpha, \theta)$ is an analytic function of $\theta$. In this section, we reproduce an argument of Avila [1] that shows that $D_{n}$ can be well approximated by a trigonometric polynomial of degree $\leq$ const $\cdot n$. This will allow to apply the estimates for trigonometric polynomials which we cite in the next section.

Let us represent $D_{n}(E, \alpha, \theta)$ by its Fourier series

$$
D_{n}(E, \alpha, \theta)=\sum_{k=-\infty}^{\infty} C_{k, n}(E, \alpha) e^{i k \theta},
$$

and let

$$
D_{n, m}(E, \alpha, \theta)=\sum_{k=-m}^{m} C_{k, n}(E, \alpha) e^{i k \theta}
$$

denote a finite piece of the Fourier series. 
Lemma 2.1. For any $R>0$, there exists $d=d(f, R)$ such that

$$
\left|D_{n}(E, \alpha, \theta)-D_{n, d n}(E, \alpha, \theta)\right| \leq e^{-n}
$$

for any $0 \leq \theta<2 \pi, \alpha \in \mathbb{R},-R \leq E \leq R$.

Proof. First we observe

$$
C_{k, n}(E, \alpha)=\frac{1}{2 \pi} \int_{0}^{2 \pi} D_{n}(E, \alpha, \theta) e^{-i k \theta} d \theta .
$$

For $k>0$, we shift the contour of integration by $-i \eta$; this yields:

$$
\begin{aligned}
\left|C_{k, n}(E, \alpha)\right| & =\left|\frac{1}{2 \pi} \int_{0}^{2 \pi} D_{n}(E, \alpha, \theta-i \eta) e^{-i k(\theta-i \eta)} d \theta\right| \\
& \leq e^{-k \eta} \max _{\theta}\left|D_{n}(E, \alpha, \theta-i \eta)\right| .
\end{aligned}
$$

Now,

$$
\begin{aligned}
\max _{\theta}\left|D_{n}(E, \alpha, \theta-i \eta)\right| & \leq 2 \max _{\theta}\left\|\left(\begin{array}{cc}
E-f(\theta-i \eta) & -1 \\
1 & 0
\end{array}\right)\right\|^{n} \\
& \leq 2\left(2+|E|+\max _{\theta}|f(\theta-i \eta)|\right)^{n} \leq(C(f)+|E|)^{n},
\end{aligned}
$$

where $C(f)$ is a positive constant depending only on $f$. Therefore

$$
\left|C_{k, n}(E, \alpha)\right| \leq e^{-k \eta}(C(f)+|E|)^{n}
$$

and

$$
\left|\sum_{k=d n}^{\infty} C_{k, n}(E, \alpha) e^{i k \theta}\right| \leq(C(f)+|E|)^{n} \frac{e^{-d n \eta}}{1-e^{-\eta}} \leq\left(C_{1}(f)+|E|\right)^{n} e^{-d n \eta} .
$$

Choosing $d$ sufficiently large, one can make this expression smaller than $e^{-n} / 2$ for $-R \leq E \leq R$. A similar argument works for $k<0$.

2.3. Estimates on trigonometric polynomials. We shall use two Remez-Turántype inequalities.

Theorem (Erdélyi [6]). Let $Q(\theta)=\sum_{k=-r}^{r} c_{k} \exp (i k \theta)$ be a trigonometric polynomial of degree $r$, and let $X \subset[0,2 \pi)$ be a measurable set, $|X| \geq 3 \pi / 2$. Then

$$
\max _{\theta \in[0,2 \pi)}|Q(\theta)| \leq e^{C r(2 \pi-|X|)} \sup _{\theta \in X}|Q(\theta)| .
$$


Theorem (Nazarov [14]). Let $Q(\theta)=\sum_{k=1}^{r} c_{k} \exp \left(i m_{k} \theta\right)$ be a trigonometric polynomial with $r$ terms, and let $X \subset[0,2 \pi)$ be a measurable set. Then

$$
\max _{\theta \in[0,2 \pi)}|Q(\theta)| \leq\left(\frac{C}{|X|}\right)^{r-1} \sup _{\theta \in X}|Q(\theta)| .
$$

Remarks. (1) The constant $C>0$ in both inequalities is universal, independent of the polynomial $Q$ under consideration.

(2) We do not use the full strength of Nazarov's theorem, even in the special case which we stated above. Indeed, the polynomials we consider are of the form $Q(\theta)=\widetilde{Q}(q \theta)$, in which case (2.2) can be derived from a version of (2.1) which covers the case $|X| \leq 3 \pi / 2$. A proof of the latter can be found for example in the work of Ganzburg [8].

(3) Similar inequalities have been previously applied to study Schrödinger operators with quasiperiodic potentials; see for example Jitomirskaya [10], Theorem 8.

\subsection{A formula for the Lyapunov exponent. Set}

$$
M_{n}(E, \alpha)=\max _{\theta}\left|D_{n}(E, \alpha, \theta)\right| .
$$

Proposition 2.2. For any $\alpha \notin \mathbb{Q}$,

$$
\bar{\gamma}(E, \alpha)=\limsup _{n \rightarrow \infty} \frac{1}{n} \ln M_{n}(E, \alpha) .
$$

Proof. First, let us show that

$$
\bar{\gamma}(E, \alpha) \leq \limsup _{n \rightarrow \infty} \frac{1}{n} \ln M_{n}(E, \alpha) .
$$

We can assume that $\bar{\gamma}(E, \alpha)>0$. Avila and Bochi [2], Theorem 15, have shown (in the general setting of ergodic $\mathrm{SL}_{2}$ sequences) that, for almost every $\theta$,

$$
\bar{\gamma}(E, \alpha)=\limsup _{n \rightarrow \infty} \frac{1}{n} \ln \rho\left(\Phi_{n}(E, \alpha, \theta)\right)
$$

(where $\rho$ stands for the spectral radius.) Recalling that

$$
\rho(\Phi) \leq|\operatorname{tr} \Phi|
$$

for $\Phi \in \mathrm{SL}_{2}(\mathbb{R})$ such that $\rho(\Phi)>1$ (which happens if and only if $|\operatorname{tr} \Phi|>2$ ), we obtain

$$
\bar{\gamma}(E, \alpha) \leq \limsup _{n \rightarrow \infty} \frac{1}{n} \ln \left|D_{n}(E, \alpha, \theta)\right| \leq \limsup _{n \rightarrow \infty} \frac{1}{n} \ln M_{n}(E, \alpha) .
$$


Let us prove the complementary inequality. Fix $\varepsilon>0$. By Egoroff's theorem, there exists $X \subset[0,2 \pi)$ such that $|X| \geq 2 \pi-\varepsilon$ and

$$
\frac{1}{n} \ln \left\|\Phi_{n}(E, \alpha, \theta)\right\| \underset{n \rightarrow \infty}{\longrightarrow} \bar{\gamma}(E, \alpha)
$$

uniformly on $X$. Therefore

$$
\limsup _{n \rightarrow \infty} \sup _{\theta \in X} \frac{1}{n} \ln \left|D_{n}(E, \alpha, \theta)\right| \leq \bar{\gamma}(E, \alpha) .
$$

Now, Erdélyi's inequality (2.1) implies

$$
\max _{\theta}\left|D_{n, d n}(E, \alpha, \theta)\right| \leq \exp (C d n \varepsilon) \sup _{\theta \in X}\left|D_{n, d n}(E, \alpha, \theta)\right|,
$$

hence by Lemma 2.1

$$
M_{n}(E, \alpha) \leq \exp (C d n \varepsilon)\left(\sup _{\theta \in X}\left|D_{n}(E, \alpha, \theta)\right|+e^{-n}\right)+e^{-n},
$$

and therefore

$$
\limsup _{n \rightarrow \infty} \frac{1}{n} \ln M_{n}(E, \alpha) \leq \bar{\gamma}(E, \alpha)+C d \varepsilon .
$$

Taking $\varepsilon \rightarrow+0$, we conclude the proof.

Now we cite several facts pertaining to (general) periodic Schrödinger operators. Let $H$ be a periodic Schrödinger operator of period $q$. The Lyapunov exponent of $H$ satisfies

$$
\gamma(E)=\lim _{n \rightarrow \infty} \frac{1}{n} \ln \left\|\Phi_{n}(E)\right\|=\frac{1}{q} \ln \rho\left(\Phi_{q}(E)\right),
$$

where $\rho$ stands for the spectral radius.

Let $E_{0}$ be an energy outside the spectrum $\sigma(H)$. Then the discriminant $\Delta$ is equal to

$$
\Delta\left(E_{0}\right)=\exp \left(q \gamma\left(E_{0}\right)\right)+\exp \left(-q \gamma\left(E_{0}\right)\right) .
$$

From the Combes-Thomas estimate (see for example [11], Theorem 11.2),

$$
\gamma\left(E_{0}\right) \geq c \min \left\{\operatorname{dist}\left(E_{0}, \sigma(H)\right), 1\right\},
$$

where $c>0$ is a universal constant. Thus we obtain the following result.

Lemma 2.3. Let $H$ be a periodic Schrödinger operator of period $q$ and let $E_{0}$ be an energy outside the spectrum $\sigma(H)$. Then

$$
\left|\Delta\left(E_{0}\right)\right| \geq \exp [c q \min \{\operatorname{dist}(E, \sigma(H)), 1\}],
$$

where $c>0$ is a universal constant. 


\section{Variations on Chambers' formula}

As before, we consider the Fourier expansion

$$
D_{n}(E, \alpha, \theta)=\sum_{k=-\infty}^{\infty} C_{k, n}(E, \alpha) \exp (i k \theta)
$$

for $D_{n}$.

In the periodic case, most of the coefficients are zero.

Proposition 3.1. For any $p / q \in \mathbb{Q}, D_{q}$ is periodic in $\theta$ of period $2 \pi / q$ :

$$
D_{q}\left(E, \frac{p}{q}, \theta+\frac{2 \pi}{q}\right)=D_{q}\left(E, \frac{p}{q}, \theta\right) .
$$

Consequently,

$$
D_{q}\left(E, \frac{p}{q}, \theta\right)=\sum_{k=-\infty}^{\infty} C_{k q, q}\left(E, \frac{p}{q}\right) \exp (i k q \theta) .
$$

Proof. First we observe

$$
V_{\frac{p}{q}, \theta+\frac{2 \pi p}{q}}(n)=V_{\frac{p}{q}, \theta}(n+1),
$$

therefore

$$
T_{n}\left(E, \frac{p}{q}, \theta+\frac{2 \pi p}{q}\right)=T_{n+1}\left(E, \frac{p}{q}, \theta\right) .
$$

Also, $T_{n}$ is a periodic sequence of period $q$, therefore

$$
\begin{aligned}
& D_{q}\left(E, \frac{p}{q}, \theta+\frac{2 \pi p}{q}\right) \\
& \quad=\operatorname{tr}\left[T_{q}\left(E, \frac{p}{q}, \theta+\frac{2 \pi p}{q}\right) \cdots T_{2}\left(E, \frac{p}{q}, \theta+\frac{2 \pi p}{q}\right) T_{1}\left(E, \frac{p}{q}, \theta+\frac{2 \pi p}{q}\right)\right] \\
& \quad=\operatorname{tr}\left[T_{1}\left(E, \frac{p}{q}, \theta\right) T_{q}\left(E, \frac{p}{q}, \theta\right) \cdots T_{2}\left(E, \frac{p}{q}, \theta\right)\right] .
\end{aligned}
$$

Since the trace is cyclic, we conclude that

$$
D_{q}\left(E, \frac{p}{q}, \theta+\frac{2 \pi p}{q}\right)=D_{q}\left(E, \frac{p}{q}, \theta\right) .
$$

Now, obviously,

$$
D_{q}\left(E, \frac{p}{q}, \theta+2 \pi\right)=D_{q}\left(E, \frac{p}{q}, \theta\right) ;
$$

since $p$ and $q$ are relatively prime, the last two equalities imply (3.2). 
The next proposition describes the leading coefficients in the trigonometric case, see (1.1).

Proposition 3.2. Suppose that the potential is defined by (1.1). For any $\alpha \in \mathbb{R}$,

$$
C_{ \pm d n, n}(E, \alpha)=\left(-a_{ \pm d}\right)^{n} \exp [ \pm \pi i \alpha d n(n+1)] .
$$

In particular, for $\alpha=p / q \in \mathbb{Q}$ and $n=q$,

$$
C_{ \pm d q, q}\left(E, \frac{p}{q}\right)=-(-1)^{(d+1)(q+1)} a_{ \pm d}^{q} .
$$

Proof. We have:

$$
C_{ \pm d n, n}=\prod_{k=1}^{n}\left\{-a_{ \pm d} \exp [ \pm 2 \pi i \alpha k d]\right\}=a_{ \pm d}^{n} \exp [ \pm \pi i \alpha d n(n+1)] .
$$

\section{Proofs of the theorems}

Bourgain and Jitomirskaya [3] have proved that the Lyapunov exponent $\bar{\gamma}(E, \alpha)$ is jointly continuous in $E$ and $\alpha$ on $\mathbb{R} \times(\mathbb{R} \backslash \mathbb{Q})$. Therefore for any $\alpha \notin \mathbb{Q}$ and $\varepsilon>0$ there exists $\delta>0$ such that for any $|p / q-\alpha|<\delta$ and any $E$ in any compact set

$$
\left|\bar{\gamma}\left(E, \frac{p}{q}\right)-\bar{\gamma}(E, \alpha)\right| \leq \varepsilon .
$$

Also observe that

$$
\begin{array}{rlr}
\bar{\gamma}\left(E, \frac{p}{q}\right) & =\lim _{n \rightarrow \infty} \frac{1}{2 \pi n} \int_{0}^{2 \pi} \ln \left\|\Phi_{n}\left(E, \frac{p}{q}, \theta\right)\right\| d \theta \\
& =\frac{1}{2 \pi} \int_{0}^{2 \pi} \gamma\left(E, \frac{p}{q}, \theta\right) d \theta \quad \text { (dominated convergence) } \\
& =\frac{1}{2 \pi q} \int_{0}^{2 \pi} \ln \rho\left(\Phi_{q}\left(E, \frac{p}{q}, \theta\right)\right) d \theta & \text { (cf. Section 2.4). }
\end{array}
$$

Now we are ready to prove the main results.

Proof of Theorem 1.1. We can assume that $\bar{\gamma}(E, \alpha)>0$. Then, for $p / q$ sufficiently close to $\alpha, \bar{\gamma}(E, p / q)>0$. Therefore there exists $\theta$ for which

$$
\frac{1}{q} \ln \rho\left(\Phi_{q}\left(E, \frac{p}{q}, \theta\right)\right)>0,
$$

that is, $E \notin \sigma(p / q, \theta)$, hence $E \notin S_{-}(p / q)$. 
Proof of Theorem 1.2. Let $\varepsilon>0$. One can find $\delta>0$ such that for any $p / q$ for which $|p / q-\alpha|<\delta$ and for any $E \in \mathcal{A}(\alpha)$ the Lyapunov exponent satisfies $\bar{\gamma}(E, p / q) \leq \varepsilon$. Therefore

$$
\varepsilon \geq \bar{\gamma}\left(E, \frac{p}{q}\right)=\frac{1}{2 \pi q} \int_{0}^{2 \pi} \ln \rho\left(\Phi_{q}\left(E, \frac{p}{q}, \theta\right)\right) d \theta,
$$

which implies

$$
\left|\left\{\theta|| D_{q}\left(E, \frac{p}{q}, \theta\right) \mid \geq 2 e^{2 q \varepsilon}\right\}\right| \leq\left|\left\{\theta \mid \rho\left(\Phi_{q}\left(E, \frac{p}{q}, \theta\right)\right) \geq e^{2 q \varepsilon}\right\}\right| \leq \pi .
$$

By Lemma 2.1,

$$
\left|\left\{\theta|| D_{q, d q}\left(E, \frac{p}{q}, \theta\right) \mid \geq 2 e^{2 q \varepsilon}+e^{-q}\right\}\right| \leq \pi .
$$

According to Proposition 3.1, $D_{q, d q}$ is a trigonometric polynomial with $2 d+1$ non-zero terms, therefore Nazarov's inequality (2.2) implies

$$
M_{q}\left(E, \frac{p}{q}\right) \leq\left(2 e^{2 q \varepsilon}+e^{-q}\right) C^{d}+e^{-q} \leq e^{2 q \varepsilon} \widetilde{C}^{d}
$$

and so for any $\theta \in[0,2 \pi)$

$$
\left|D_{q}\left(E, \frac{p}{q}, \theta\right)\right| \leq e^{2 q \varepsilon} \widetilde{C}^{d} .
$$

On the other hand, Lemma 2.3 tells us that for energies $E$ at distance $\tilde{\varepsilon}>0$ from $\sigma(p / q, \theta)$,

$$
\left|D_{q}\left(E, \frac{p}{q}, \theta\right)\right| \geq \exp (c q \min (\tilde{\varepsilon}, 1)) .
$$

Therefore $\tilde{\varepsilon} \leq C \varepsilon$, hence

$$
\operatorname{dist}\left(E, \sigma\left(\frac{p}{q}, \theta\right)\right) \leq C \varepsilon .
$$

This is true for any $\theta$, hence

$$
E \in S_{-}\left(\frac{p}{q}, C \varepsilon\right) .
$$

We have shown that

$$
\mathcal{A}(\alpha) \subset S_{-}\left(\frac{p}{q}, C \varepsilon\right) ;
$$

taking the intersection over all $\varepsilon>0$, we conclude the proof.

To prove Theorem 1.3, we need a general statement. 
Proposition 4.1. For any $p / q \in \mathbb{Q}$ and $E \in \mathbb{R}$,

$$
\left|\left\{\theta \in[0,2 \pi) \mid E \in \sigma\left(\frac{p}{q}, \theta\right)\right\}\right| \leq C M_{q}\left(E, \frac{p}{q}\right)^{-\frac{1}{2 d}},
$$

where $d=d(f)$ depends only on $f$. In the trigonometric case (1.1), one may take $d$ to be the degree of $P$.

Proof. For simplicity, we prove the proposition in the trigonometric case (1.1); the general case follows as before from the approximation of Lemma 2.1.

Denote

$$
\Upsilon\left(E, \frac{p}{q}\right)=\left\{\theta \in[0,2 \pi) \mid E \in \sigma\left(\frac{p}{q}, \theta\right)\right\} .
$$

By Proposition 3.1, $D_{q}(E, p / q, \cdot)$ is a trigonometric polynomial with $2 d+1$ terms. Therefore, by Nazarov's inequality (2.2) and the fact that the (essential) spectrum of a periodic operator is the inverse image of $[-2,2]$ under the discriminant,

$$
M_{q}\left(E, \frac{p}{q}\right) \leq\left[\frac{C}{\left|\Upsilon\left(E, \frac{p}{q}\right)\right|}\right]^{2 d} \max _{\theta \in \Upsilon\left(E, \frac{p}{q}\right)}\left|D_{q}\left(E, \frac{p}{q}, \theta\right)\right| \leq 2\left[\frac{C}{\left|\Upsilon\left(E, \frac{p}{q}\right)\right|}\right]^{2 d}
$$

and hence

$$
\left|\Upsilon\left(E, \frac{p}{q}\right)\right| \leq C M_{q}\left(E, \frac{p}{q}\right)^{-\frac{1}{2 d}}
$$

(with a different constant $C>0$ ).

Proof of Theorem 1.3. Let $\varepsilon>0$. Choose $\delta>0$ such that for any energy $E$ and any $p / q$ such that $|p / q-\alpha|<\delta$ and

$$
\bar{\gamma}\left(E, \frac{p}{q}\right) \geq \bar{\gamma}(E, \alpha)-\varepsilon .
$$

If $\bar{\gamma}(E, \alpha)>\varepsilon$,

$$
\begin{aligned}
\bar{\gamma}(E, \alpha)-\varepsilon \leq \bar{\gamma}\left(E, \frac{p}{q}\right) & =\frac{1}{2 \pi q} \int_{0}^{2 \pi} \ln \rho\left(\Phi_{q}\left(E, \frac{p}{q}, \theta\right)\right) d \theta \\
& \leq \max _{\theta} \frac{1}{q} \ln \rho\left(\Phi_{q}\left(E, \frac{p}{q}, \theta\right)\right) \leq \frac{1}{q} \ln M_{q}\left(E, \frac{p}{q}\right) .
\end{aligned}
$$

Therefore

$$
M_{q}\left(E, \frac{p}{q}\right) \geq \exp [q(\bar{\gamma}(E, \alpha)-\varepsilon)] .
$$

Combining this with the previous proposition, we obtain the estimate.

Corollary (1.2) follows from the Fubini theorem. 


\section{Herman's inequality}

The results of this section pertain to the trigonometric case (1.1).

Proposition 5.1. For any $\alpha \notin \mathbb{Q}$ and any $E \in \mathbb{R}$,

$$
\bar{\gamma}(E, \alpha) \geq \ln _{+}\left|a_{d}\right| .
$$

Proof. By Proposition 2.2,

$$
\bar{\gamma}(E, \alpha)=\limsup _{n \rightarrow \infty} \frac{1}{n} \ln M_{n}(E, \alpha) \geq \limsup _{n \rightarrow \infty} \frac{1}{2 n} \ln \frac{1}{2 \pi} \int_{0}^{2 \pi}\left|D_{n}(E, \alpha, \theta)\right|^{2} d \theta .
$$

According to Proposition 3.2 we deduce

$$
\bar{\gamma}(E, \alpha) \geq \limsup _{n \rightarrow \infty} \frac{1}{n} \ln \left|C_{d n, n}(E)\right|=\ln \left|a_{d}\right| .
$$

For the periodic case, one has the following result.

Proposition 5.2. If $\left|a_{d}\right|>1, S_{-}\left(\frac{p}{q}\right)=\varnothing$ for $q>\frac{1}{2 \log _{2}\left|a_{d}\right|}$.

Proof. By Proposition 3.2,

$$
M_{q}\left(E, \frac{p}{q}\right) \geq\left[\frac{1}{2 \pi} \int_{0}^{2 \pi}\left|D_{q}\left(E, \frac{p}{q}, \theta\right)\right|^{2} d \theta\right]^{1 / 2} \geq \sqrt{2}\left|a_{d}\right|^{q},
$$

which is larger than 2 for $q>\frac{1}{2 \log _{2}\left|a_{d}\right|}$.

\section{References}

[1] A. Avila, Global theory of one-frequency Schrödinger operators I: stratified analyticity of the Lyapunov exponent and the boundary of nonuniform hyperbolicity. Preprint 2009 arXiv:0905.3902

[2] A. Avila and J. Bochi, A formula with some applications to the theory of Lyapunov exponents. Isr. J. Math. 131 (2002), 125-137. MR 1942304 Zbl 1022.37019

[3] J. Bourgain and S. Jitomirskaya, Continuity of the Lyapunov exponent for quasiperiodic operators with analytic potential, Dedicated to David Ruelle and Yasha Sinai on the occasion of their 65th birthdays. J. Stat. Phys. 108 (2002), 1203-1218. MR 1933451 Zbl 1039.81019

[4] W. Chambers, Linear network model for magnetic breakdown in two dimensions. Phys. Rev. A 140 (1965), 135-143.

[5] H. L. Cycon, R. G. Froese, W. Kirsch, and B. Simon, Schrödinger operators with application to quantum mechanics and global geometry. Springer Verlag, Berlin etc., 1987. MR 0883643 Zbl 0619.47005 
[6] T. Erdélyi, Remez-type inequalities on the size of generalized polynomials. J. Lond. Math. Soc., II. Ser. 45 (1992), 255-264. MR 1171553 Zbl 0712.41002

[7] H. Furstenberg and H. Kesten, Products of random matrices. Ann. Math. Stat. 31 (1960), 457-469. MR 0121828 Zbl 137.35501

[8] M. I. Ganzburg, Polynomial inequalities on measurable sets and their applications, Constr. Approx. 17 (2001), 275-306. MR 1814358 Zbl 0991.41008

[9] M. Herman, Une méthode pour minorer les exposants de Lyapounov et quelques exemples montrant le caractère local d'un théorème d'Arnol'd et de Moser sur le tore de dimension 2, Comment. Math. Helv. 58 (1983), 453-502. MR 0727713 Zbl 0554.58034

[10] S. Jitomirskaya, Metal-insulator transition for the almost Mathieu operator, Ann. of Math. (2) 150 (1999), 1159-1175. MR 1740982 Zbl 0946.47018

[11] W. Kirsch, An invitation to random Schrödinger operators. Soc. Math. France, Paris, 2008. With an appendix by F. Klopp. MR 2509110 Zbl 1162.82004

[12] Y. Last, A relation between a.c. spectrum of ergodic Jacobi matrices and the spectra of periodic approximants, Commun. Math. Phys. 151 (1993), 183-192. MR 1201659 Zbl 0782.34084

[13] Y. Last, Spectral theory of Sturm-Liouville operators on infinite intervals: a review of recent developments. In Amrein, Werner O. (ed.) et al., Sturm-Liouville theory. Past and present. Selected survey articles based on lectures presented at a colloquium and workshop in Geneva, Italy, September 15-19, 2003 to commemorate the 200th anniversary of the birth of Charles François Sturm., Birkhäuser, Basel, 2005, 99-120. MR 2145079 Zbl 1098.39011

[14] F. L. Nazarov, Local estimates of exponential polynomials and their applications to inequalities of uncertainly principle type. Algebra Anal. 5 (1993), 3-66. English transl.: St. Petersburg Math. J. 5 (1994), 663-717. MR 1246419 Zbl 0801.42001

Received March 3, 2011; revised April 17, 2011

Mira Shamis, School of Mathematics, Institute for Advanced Study, Einstein Dr., Princeton, NJ 08540, U.S.A.

E-mail: shamis@ias.edu 